\title{
Inferior mesenteric arteriovenous fistula
}

\author{
Seunghun Lee, Jooweon Chung, Byungkwon Ahn, Seunghyun Lee, Sunguhn Baek \\ Division of Colorectal Surgery, Kosin University Gospel Hospital, Kosin University College of Medicine, Busan, Korea
}

\begin{abstract}
Arteriovenous fistula (AVF) involving the inferior mesenteric artery and vein is very rare with only 33 cases described in the literature and may be of congenital or acquired (iatrogenic or traumatic) or idiopathic etiology. The pathophysiology of AVF that acts as a left-to-right shunt has accounted for clinical signs and symptoms associated with ischemic colitis, portal hypertension, and heart failure. A low incidence and nonspecific clinical signs and symptoms such as abdominal pain, thrill and mass, lower and upper gastrointestinal bleeding make it difficult to establish a diagnosis of inferior mesenteric AVF. Diagnosis of inferior mesenteric AVF is usually established by radiological or intraoperative examination. We report a case of idiopathic inferior mesenteric AVF causing ischemic colitis in a 56-year-old man that was diagnosed preoperatively by multidetector computed tomography and angiography and successfully treated by surgical resection.
\end{abstract}

[Ann Surg Treat Res 2017;93(4):225-228]

Key Words: Arteriovenous fistula, Inferior mesenteric artery, Ischemic colitis, Portal hypertension

\section{INTRODUCTION}

An arteriovenous fistula (AVF) is an abnormal communication or passageway between an artery and a vein, bypassing the capillary bed. The AVFs can be of congenital or acquired (iatrogenic or traumatic) or idiopathic etiology [1-7]. Congenital AVFs occur from undifferentiated embryonic vessels that fail to differentiate into arteries and veins. The rupture of a congenital arterial aneurysm very close to a vein can also result in the formation of an AVF [8]. Iatrogenic AVFs between inferior mesenteric artery (IMA) and vein (IMV) can occur after arterial catheterization or various surgical procedures such as a sigmoidectomy or left hemicolectomy $[1,6]$. AVFs can occur as a result of blunt or penetrating traumas such as a gunshot or stab wound [1,7] or idiopathic etiology [3-5]. Although more than 200 cases of AVFs between the hepatic, splenic, and superior mesenteric vessels have been reported in the literature, AVF involving the inferior mesenteric artery and vein (IMAV AVF) is very rare [1-7]. We report a case of idiopathic IMAV AVF with ischemic colitis treated with surgical excision.

\section{CASE REPORT}

A 56-year-old man presented to Kosin University Gospel Hospital with recurrent lower abdominal pain, watery diarrhea and hematochezia of 3 months. Before this, the patient was in good health without underlying disease. The patient could not recall previous similar symptoms and had no history of previous abdominal surgery or trauma. Abdominal examination revealed tenderness in the left lower quadrant and decreased bowel sounds. The digital rectal examination was unremarkable. There was no clinical evidence of portal hypertension.

Laboratory tests were unremarkable except $120 \mathrm{~mm} / \mathrm{hr}$ erythrocyte sedimentation rate (range, 0-16) and $3.3 \mathrm{mg} / \mathrm{dL}$ highsensitivity C-reactive protein (range, $0-0.75$ ). Colonoscopy showed abnormal mucosa associated with diffuse distortion of vascular pattern, coarse granularity and friability with
Received November 17, 2016, Revised January 23, 2017 ,

Accepted February 8, 2017

Corresponding Author: Byungkwon Ahn

Division of Colorectal Surgery, Kosin University Gospel Hospital, Kosin University College of Medicine, 262 Gamcheon-ro, Seo-gu, Busan 49267, Korea

Tel: +82-51-990-6462, Fax: +82-51-246-6093

E-mail: gsabk@hotmail.com
Copyright (c) 2017, the Korean Surgical Society

(c) Annals of Surgical Treatment and Research is an Open Access Journal. All articles are distributed under the terms of the Creative Commons Attribution NonCommercial License (http://creativecommons.org/licenses/by-nc/4.0/) which permits unrestricted non-commercial use, distribution, and reproduction in any medium, provided the original work is properly cited. 

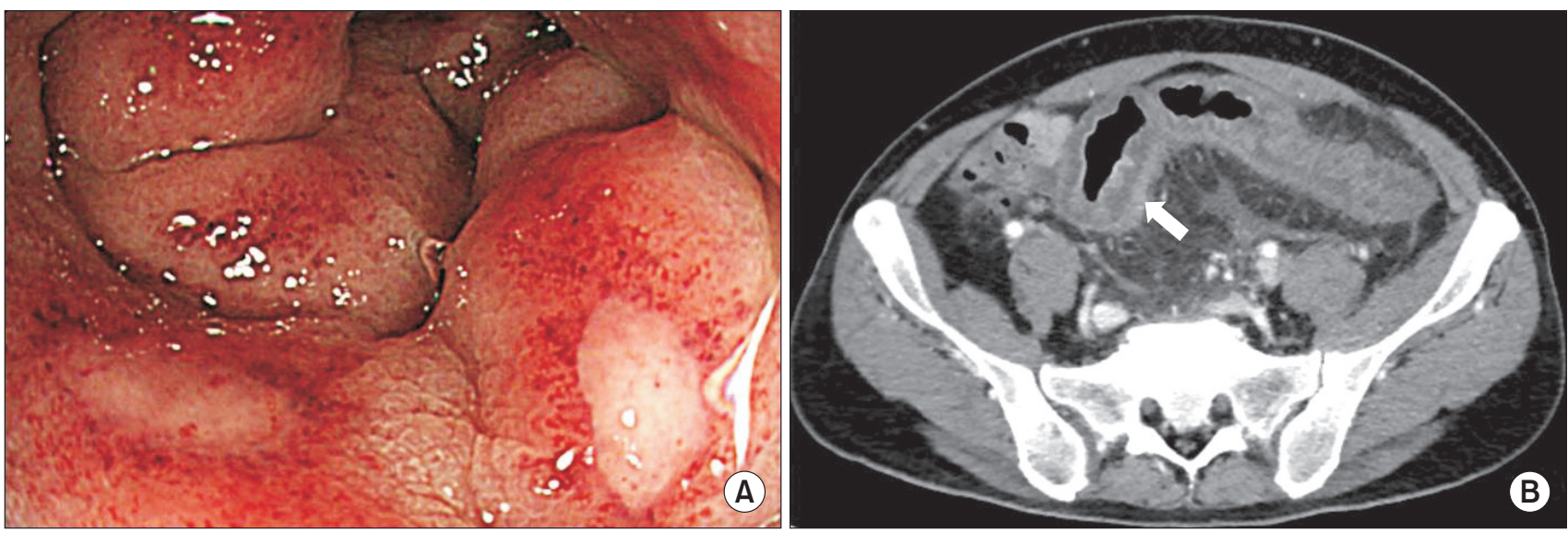

Fig. 1. (A) Colonoscopy shows abnormal colonic mucosa with multiple ulcer, exudate and hemorrhage from the splenic flexure to the upper rectum. (B) CT scan (axial view) reveals edematous, thickened and hypoenhanced wall of sigmoid colon with adjacent fat stranding (arrow) in arterial phase.

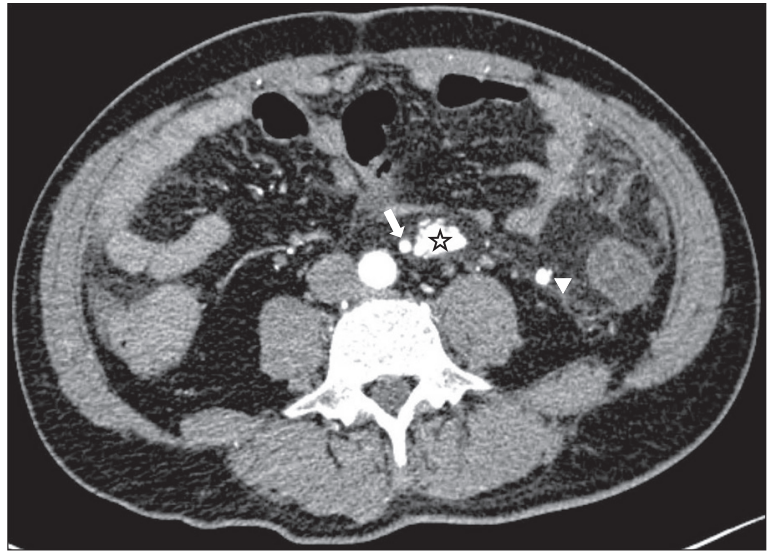

Fig. 2. Multidetector computed tomographic angiography (axial image) shows an arteriovenous communication (star) supplied by dilated inferior mesenteric artery (arrow) with venous drainage into dilated marginal vein (arrowhead).

focal asymmetrical patchy discontinuous pattern from the splenic flexure to the upper rectum. At the sigmoid colon, 28 $\mathrm{cm}$ proximal from the anal verge, multiple-sized round ulcers with whitish exudate covering were noted (Fig. 1A). CT scan revealed diffuse wall thickening and decreased enhancement of descending, sigmoid colon and rectum, most apparent at the sigmoid colon (Fig. 1B). An evaluation of infectious etiologies was negative. Multidetector computed tomographic (MDCT) angiography was performed with suspicion of mesenteric ischemia. MDCT angiography demonstrated abnormal serpentine artery branching pattern supplied by an enlarged IMA and occlusion of the main branch of the IMV with venous drainage into the splenic and superior mesenteric vein (SMV) via a markedly dilated marginal vein in the arterial phase (Figs. 2, 3). An angiography of the IMA was performed for a definite diagnosis and embolization. Angiography showed nidus of the IMAV

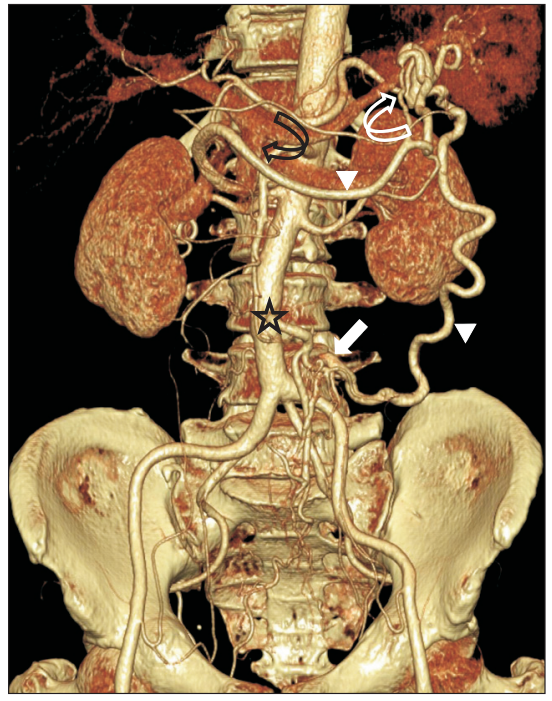

Fig. 3. Multidetector computed tomographic angiography (3-dimensional volume-rendered image) shows enlarged fistulous communication (arrow) supplied from inferior mesenteric artery (star) with venous drainage into splenic (white curved arrow) and superior mesenteric vein (black curved arrow) via marginal vein (arrow heads).

AVF supplied by multiple small serpentine arteries originate from the superior and inferior division of IMA with a single dominant draining vein into SMV via the marginal vein (Fig. 4). Because of its large size $(>8 \mathrm{~mm})$, high flow rate, multiple feeding vessels of AVF, and risk of organ ischemia, surgical excision was performed instead of angiographic embolization.

Exploratory laparoscopy was performed and converted to an open operation due to the extensively inflamed and thickened mesentery of the left colon. Resection consisting of the descending, sigmoid colon and upper rectum was performed. An end colostomy was created. Ischemic colitis and 

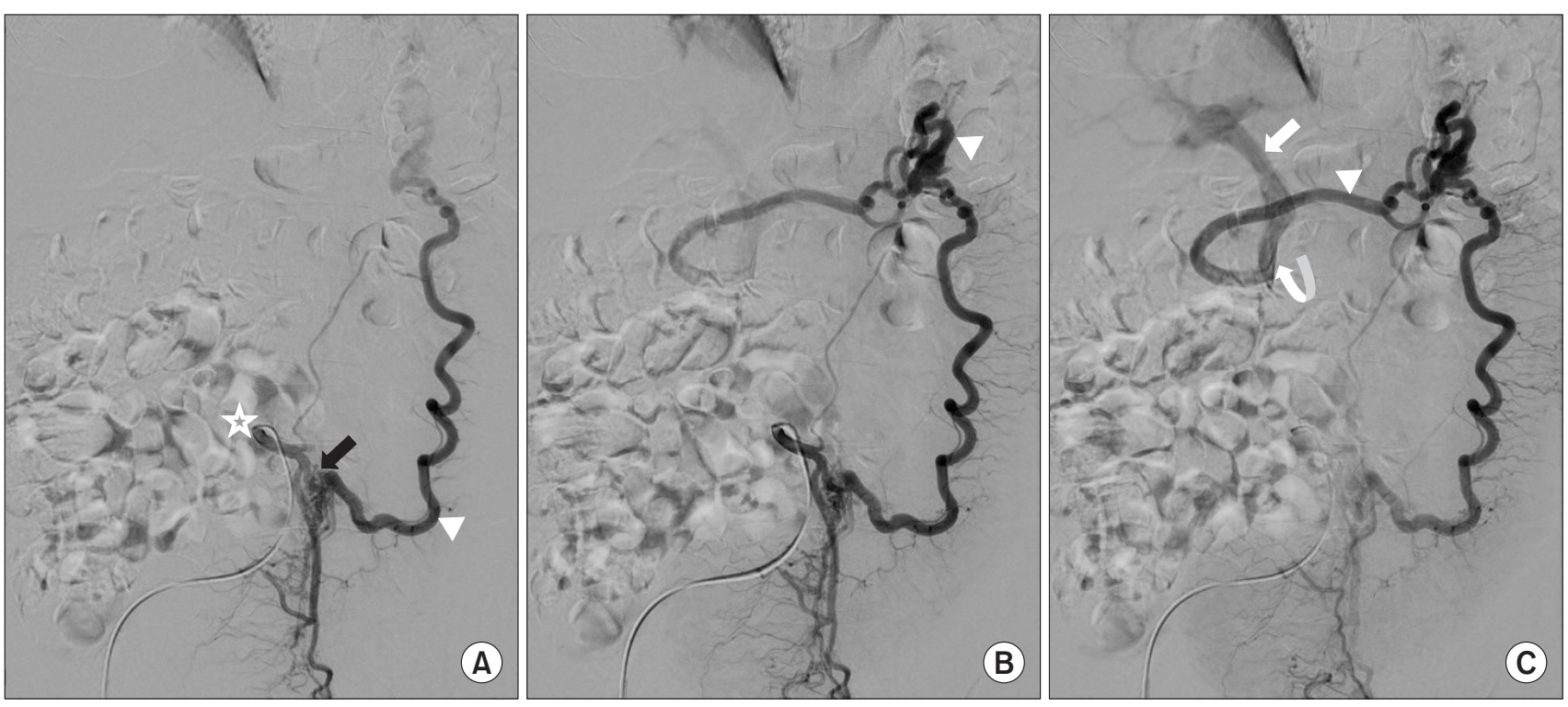

Fig. 4. Early $(\mathrm{A})$, middle $(\mathrm{B})$, late $(\mathrm{C})$ arterial phase images from angiography of the inferior mesenteric artery shows nidus of fistula (black arrow) supplied by inferior mesenteric artery (star). There is prompt filling of dilated marginal vein (arrow head) with drainage into splenic, superior mesenteric (curved arrow) and portal vein (white arrow). The inferior mesenteric vein is occluded.

AVF were confirmed in pathology. The postoperative course was uneventful and the patient was discharged on the eighth postoperative day. The colostomy was restored one year after surgery, and repeated colonoscopy and CT scan were unremarkable.

The patient signed informed consent forms previously approved by the institutional review board of the Kosin University Gospel Hospital.

\section{DISCUSSION}

IMAV AVFs are rare, 33 cases have been reported in the literature for about a half-century [1-7]. Among the reported cases, 20 cases were congenital or idiopathic [1-5] and the rest were usually secondary to previous operation or trauma $[1,6,7,9]$.

An AVF is usually associated with decreased arterial blood flow to the tissue beyond the fistula and increased venous pressure distal to the fistula [10]. Consequently, colonic ischemia can result from hypoperfusion of the mucosa secondary to a steal phenomenon, with blood flow through the AVFs bypassing the capillary bed of the colon. Venous hypertension can also facilitate colonic ischemia with congestion and edema of the mucosa. Shunting of arterial flow from the IMV to the portal system can cause portal hypertension that may precipitate from both an increase in blood flow into the portal system and a compensatory increase in hepatic vascular resistance [10]. Portal hypertension is reported to occur in approximately $50 \%$ of patients with splanchnic AVFs [8,10]. Okada et al. [9] reported that 7 of 11 patients with IMAV AVF had either signs or symptoms of portal hypertension or elevated portal venous pressure. In the current case, the patient had no evidence of portal hypertension on physical examination, laboratory tests and imaging study. The pathophysiology of AVF that acts as a left-to-right shunt has accounted for clinical symptoms and signs such as abdominal pain, thrill and mass, lower and upper gastrointestinal bleeding, ischemic colitis, portal hypertension, and heart failure $[1,10]$.

Mesenteric AVFs are usually detected within weeks of the initial injury, although delays in diagnosis of up to several years have been reported [7]. It took three months from symptoms to diagnosis in this case. The patient is in his middle fifties and had no medical history of abdominal surgery or injury. It seemed reasonable to assume that the fistula originated from idiopathic etiology. Low incidence and nonspecific clinical signs and symptoms make it difficult to establish a diagnosis of IMAV AVFs. A medical history of an operation or trauma, the clinical signs and symptoms of ischemic colon, identification of AVFs by imaging study such as MDCT angiography or angiography of the IMA, and intraoperative finding of a congested colon are necessary for definitive diagnosis.

Potential treatment of IMAV AVFs is surgical correction of the AVFs with or without bowel resection. Another choice is percutaneous endovascular embolization of the feeding artery [10]. In this case, percutaneous endovascular embolization was thought to include the risks of distal migration of embolic agents, occlusion of an important source of blood supply to the colon, and partial success with embolization due to multiple feeding vessels of AVFs [10]; so we decided on surgery. 
Idiopathic IMAV AVFs without portal hypertension are extremely rare. We suggest that with clinical symptoms and signs of ischemic colitis without a reliable etiology, mesenteric AVFs should be considered. Appropriate treatment based on high clinical suspicion can reduce the risk of complications and postoperative morbidity and mortality of this disease entity.

\section{CONFLICTS OF INTEREST}

No potential conflict of interest relevant to this article was reported.

\section{REFERENCES}

1. Athanasiou A, Michalinos A, Alexandrou A, Georgopoulos S, Felekouras E. Inferior mesenteric arteriovenous fistula: case report and world-literature review. World J Gastroenterol 2014:20:8298-303.

2. Justaniah AI, Molgaard C, Flacke S, Barto A, Iqbal S. Congenital inferior mesenteric arteriovenous malformation presenting with ischemic colitis: endovascular treatment. J Vasc Interv Radiol 2013;24:1761-3.

3. Hendy P, Cheng EH, Livsey R, Mortimore $M$. Curative embolization of an inferior mesenteric arteriovenous fistula causing ischaemic proctosigmoiditis. ANZ J Surg 2015 Oct 13 [Epub]. https://doi.org/10.1111/ ans.13346.
4. Faghihi Langroudi T, Shabestari AA, Pourghorban R, Pourghorban R. Idiopathic inferior mesenteric arteriovenous fistula: a rare cause of pulsatile abdominal mass. Indian J Surg 2015;77(Suppl 1):84-6.

5. Noor M, Cooper K, Lujan H, Pena C. Arteriovenous malformation of the inferior mesenteric artery presenting as ischemic colitis. Vasc Med 2016;21:555-7.

6. Carbonell S, Ortiz S, Enriquez P, Lluis F. Arteriovenous fistula in the inferior mesenteric territory. Cir Esp 2014:92:e23.

7. Jeon DO, Park JS, Kim JE, Lee SJ, Cho HJ, Im SG, et al. A case of traumatic inferior mesenteric arteriovenous fistula. Korean J Gastroenterol 2013;62:296-300.
8. Van Way CW 3rd, Crane JM, Riddell DH, Foster JH. Arteriovenous fistula in the portal circulation. Surgery 1971;70:876-90.

9. Okada K, Furusyo N, Sawayama Y, Ishikawa N, Nabeshima S, Tsuchihashi T, et al. Inferior mesenteric arteriovenous fistula eight years after sigmoidectomy. Intern Med 2002;41:543-8.

10. Capron JP, Gineston JL, Remond A, Lallement PY, Delamarre J, Revert R, et al. Inferior mesenteric arteriovenous fistula associated with portal hypertension and acute ischemic colitis. Successful occlusion by intraarterial embolization with steel coils. Gastroenterology 1984;86:351-5. 